\title{
Toward a Learning/Instruction Process Model for Facilitating the Instructional Design Cycle
}

\author{
Yusuke Hayashi ${ }^{1}$, Seiji Isotani ${ }^{1}$, Jacqueline Bourdeau ${ }^{2}$, \\ and Riichiro Mizoguchi ${ }^{1}$ \\ ${ }^{1}$ ISIR, Osaka University, Japan \\ \{hayashi,isotani,miz\}@ei.sanken.osaka-u.ac.jp \\ ${ }^{2}$ LICEF Research Center, TÉLUQ-UQAM, Canada \\ jacqueline.bourdeau@licef.ca
}

\begin{abstract}
Many instructional design models have been proposed and their benefits are evident. However, there is lack of a common and formal notation to describe the product of the design. This causes difficulty in evaluating the product (the course) in the development. To eliminate the difficulty, we need a formal framework which has enough semantics for keeping the consistency of the product. Thus, this work aims at proposing a unified modeling framework for learning and instruction based on ontologies that has the potential to support some phases of instructional design. Furthermore, we give an example of how one-to-one instruction and collaborative learning are modeled on the proposed framework.
\end{abstract}

Keywords: Ontological engineering, instructional design, collaborative learning.

\section{Introduction}

A considerable number of instructional design (ID) models have been proposed. The main contribution of them is to provide systematic and reflective processes for developing learning/instructional courses. All of these process models share most of the same basic components: analysis, design, development, implementation, and evaluation [17]. Each component has a discipline for an assessment of the course in bringing about learning and a mechanism to improve the course if learning fails to occur as expected. Therefore the final product of ID (learning/instructional process description as a course) can be modified until it reaches the desired quality level [5].

In order to go through the whole ID process, it is necessary to ensure the consistency of the product of each phase across the overall process. However, there is (still) no real tradition in education of making formal notations of course designs. Such lack of common and formal notations makes the course development very local which hampers broader sharing between ID phases or stakeholders and impedes a better evaluation of design products [16].

To establish a common and formal notation, development and use of EMLs [20] and scripts $[7,10]$ have been moderately adopted by the community. Currently EMLs are integrated into IMS learning design (LD) specification as a standard [11] providing a sufficiently flexible framework that can be used to describe formally the design 
of almost any teaching-learning process [16]. Although such approach is much better than free handwriting notations, it neither helps users to keep the consistency/validity of the course throughout the ID process nor allows for the development of intelligent tools that can support users during the design process.

Thus, the final goal of this study is to establish a comprehensive model for describing formally the design of variety forms of learning/instruction ${ }^{1}$ (e.g. those summarized in [21]) through ontological engineering approach [3, 4, 18]. Especially, in this paper, we discuss a unification of one-to-one instruction, such as tutoring or individual e-learning course, and collaborative learning, in which learners teach and learn from each other. Although the attention to blended learning has been growing, most of the studies have been made on either type of them. Such a unified model will contribute to expansion of the range of instructional design and to share the design rationale of a course through the overall ID phases. Ontological engineering is expected to provide guidelines to find out the key concepts for such a unified model. In addition, while it cannot be discussed in this paper in detail, such a model is also expected to make contributions to modeling instructional design knowledge, which provides a valid composition of a model.

This paper is organized as follows: In section 2, instructional design processes are summarized and the requirements for comprehensive learning/instructional design process management are discussed together with its overview. In section 3, we describe ontologies we have proposed as the basis for a comprehensive model that support various forms of learning and instruction. The fourth section presents an example of modeling collaborative learning based on the Peer Tutoring theory. Finally, we conclude this paper with future directions of this study.

\section{Towards a Comprehensive ID Process Management}

This section gives an overview of the main phases of the available ID process models and discusses the requirement for comprehensive learning/instructional design process management. As mentioned in section 1, all ID process models share most of the same basic phases: The analysis phase involves analyzing a specific educational problem. The product of this phase is the terminal objective of the course. Usually, a list of questions is used to conduct analysis and the results are described narratively or in informal diagrams. In the design phase, learning/ instructional strategies to achieve the terminal objective are identified. The main product of this phase is a flow of learning/instruction which works as the mold for a particular learning/instruction. In the development phase, specific learning/ instructional materials used in the execution are assigned to the product of the design phase. In the implementation phase the course is delivered to learners and learning is conducted by it. The output of this phase is actual data of learning conducted by the course. Finally, in the evaluation phase, data collected in the implementation phase are compared with the design of the course. The gap between them is the point to be improved in the current course. Based on this result, the ID process returns to any other phase for improvement.

\footnotetext{
${ }^{1}$ The term "instruction" is used in the wider sense in this paper therefore this means not only what a person does to instruct others but also what one does to support or facilitate learning of others [2]. The term "instructor" is also used in the sense.
} 
Through these phases, a course is produced as the final product that reaches the desired quality level. The problem pointed out here is that most of the products of each process are managed with narrative or simple, non-formal diagrams and tables [22]. Although IMS LD provides a formal framework to describe the products, this is just a format and does not have enough semantics for keeping their consistency or for assessing their validity [1].

This study proposes a framework to model the product (course) to manage the input and the output of each phase in the ID process comprehensively. If the framework has the potential to describe any learning/instruction process from the learning objective of a course to the learning materials employed in the course, the product can be maintained across the ID process consistently.

We take the ontological engineering approach to tackle this issue through defining concepts related learning and instruction and organizing them as an ontology based on philosophical considerations. Figure 1 draws a rough sketch of a learn-

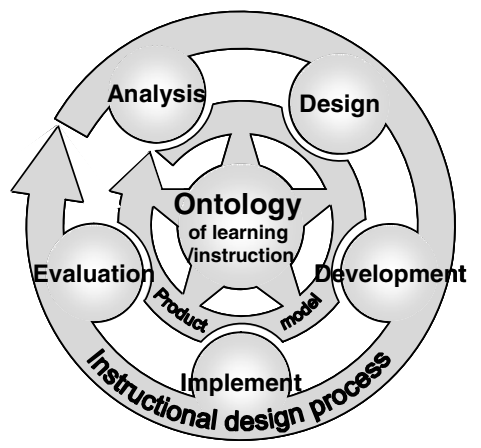

Fig. 1. ID process and ontology ing/instructional process model for facilitating the ID cycle based on such an ontology. The center of the figure denotes an ontology that defines concepts for modeling learning and instruction process as the product. The cycle around the ontology is the instructional design process composed of the typical basic phases. The ontology will be a foundation for maintenance of the product throughout the ID process. Currently the focus of this study is mainly on the input and output of the design phase (and a part of the development phase).

\section{Ontologies for Modeling Learning and Instruction}

We have proposed two ontologies for modeling learning: OMNIBUS [18, 19] and the Collaborative Learning (CL) ontology $[12,15]$. Although the target of the former is one-to-one/more instruction and the latter is collaborative learning, both of them are based on the same working hypothesis and aim at providing a conceptual framework to model learning and instruction as well as structuring learning/instructional theories as guidelines to compose good learning and instructional scenarios. The core idea of these ontologies is that "learning" can be modeled as state change of learners. This is based on our working hypothesis that a sharable "engineering approximation" of the concept "learning" can be found in terms of the changes that are taking place in the state of the learners [8].

This core idea is conceptualized as $I \_L$ event and shared by the two ontologies. This concept, in which "I_L" stands for the relationship between Instruction and Learning, describes a learner state is achieved by the learner's action affected by the other's action, which can be considered to have any instructional effect. Under the concept of I_L event, the relationships among the actions and the learner's state change are conceptualized as one. This makes it possible to describe the relationships among various learning/instructional actions and state changes. 
The following sub-sections describe, briefly, how individual learning and collaborative learning is modeled with I_L event in the two ontologies as the basis for a comprehensive modeling framework for the instructional design process.

\subsection{OMNIBUS}

One of the characteristics of OMNIBUS is to model learning/instructional process at various levels of granularity. At each level of granularity, learning/ instruction process is modeled as a sequence of I_L event and the levels are multi-layered. In the layers, each I_L event at the upper level is related to I_L events at the lower one. This relation offers both top-down and bottom-down interpretations; the lower state changes of learner achieve the upper one and the upper action is realized by the lower ones, respectively. In OMNIBUS this is conceptualized as "WAY" In short, I_L events describe what to achieve and WAYs describe how to achieve it. Fig. 2 (a) shows an example of WAY. In the Fig. 2 (a), the oval nodes represent I_L events, and black squares linking the

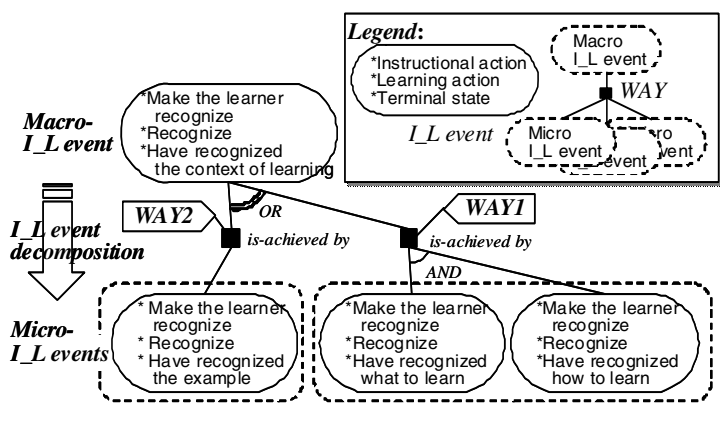

(a) A basic unit of a scenario model

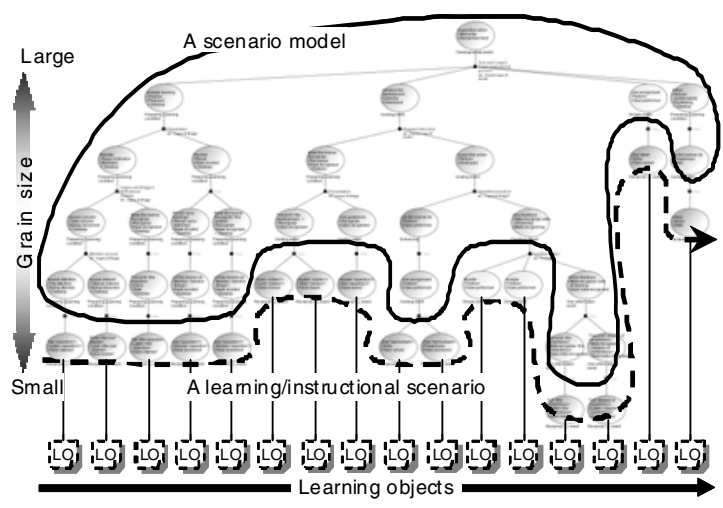

(b) An example of scenario model

Fig. 2. Scenario modeling based on OMNIBUS macro and the micro I_L events represent WAYs. Here, the macro I_L event has two WAYs; WAY1 and WAY2, and there is an "OR" relation between them. This indicates that there are two alternatives to achieve the macro I_L event.

Based on OMNIBUS, a learning/instructional scenario is modeled described as a tree structure of I_L events decomposed by WAYs as shown in Fig. 2 (b). The leaf layer is a description of a learning/instructional scenario executed by instructors and learners, and is linked with LOs used in the execution. The tree structure excepting the leaf level explains the design rationale of the scenario and it works as the specifications of the LOs to be attached. 
These concepts of I_L event and WAY also give a conceptual scheme to model strategies from learning/instructional theories. We have extracted 99 strategies from 11 theories and defined them as WAYs in OMNIBUS [9]. Such WAYs based on learning/instructional design knowledge, which includes learning/instructional theories, patterns and best practices, are called "WAY-knowledge" in OMNIBUS. This WAY-knowledge works as the guidelines for designing scenarios and as a justification to demonstrate their validity.

\subsection{Collaborative Learning (CL) Ontology}

The focal points of the CL ontology are also state changes, which are "learning", of each participant in collaborative learning and interactions between them. These are modeled as Growth Model Improved by Interaction Patterns (GMIP) [15] employing I_L event. Figure 3 shows an example of GMIP. GMIP has two components: one is Learner Growth Model (LGM) [13] and the other is Interaction pattern (IP) [14]. As shown in Figure 3(a), LGM represents, in a simplified way, possible transitions of states in the learner's knowledge acquisition process and skill development process as links in the graph.

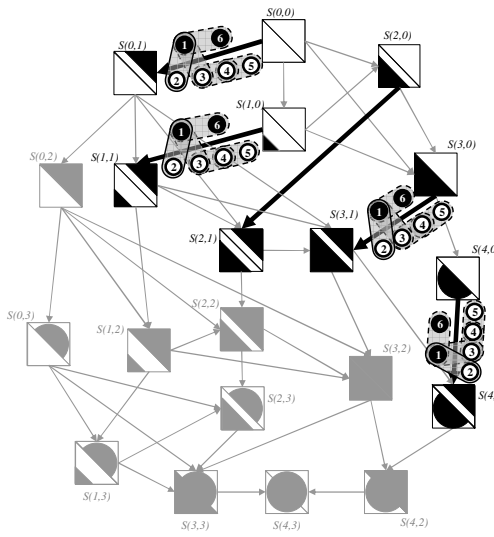

(A) The LGM for Peer tutoring: Learning by being taught (Peer tutee)

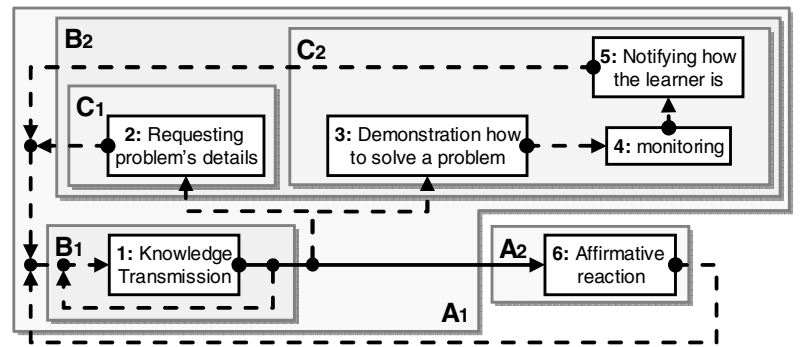

(B) The Interaction pattern for Peer tutoring

Fig. 3. Growth Model Improved by Interaction Patterns (GMIP) IP represents the flow of interaction between learners as shown in Figure 3(b), in which a node denotes an interaction modeled as I_L event. Through the connection of LGM and IP in GMIP each transition between states is connected with interactions between participants.

In collaborative learning, each participant is a learner with his/her own learning objective and sometimes his/her action helps or facilitates learning of others, which is referred to as instructional action in the conceptualization of I_L event. For example, in the theory of "Peer tutoring" [6], two types of role are defined: PeerTutor-role and PeerTutee-role. Participants assigned to a PeerTutee-role (PeerTutees) learn through 
being taught by the others assigned to a PeerTutee-role (PeerTutors). And the PeerTutors also learn through teaching the PeerTutees. The important point here is that from the point of view of CL the PeerTutor does not act as a real instructor, who only teaches, because he/she is also a learner through learning by teaching. Such a dualnature of a participant can be modeled by I_L events. Focusing on learning in PeerTutee-role, when a PeerTutee learns, a PeerTutor support the PeerTutee by teaching. On the other hand, focusing on learning in PeerTutor-role, when a PeerTutor learns, a PeerTutee support the learning by being taught. These are described in two different I_L events. Thus, in an I_L event, the PeerTutor teaches the PeerTutee, and, in another I_L event, he/she learns through teaching the PeerTutee.

GMIP defines one IP and one or more LGMs corresponding to each role. Thus, although Fig. 3 has only one LGM for PeerTutee-role, actually there is another LGM for PeerTutor-role. GMIP helps to explicitly show how learners in the group should interact with each other and the benefits for learners playing different roles. Thus, it becomes a powerful tool in helping designers to select appropriate interactions and roles to achieve desired learning goals.

\section{An Integrated Model of Learning and Instruction}

Based on the ontologies described in the previous section, we aim at modeling various forms of learning/instruction (e.g. those summarized in [21]), which is the product of the ID process. As discussed previously, employing I_L event as the basis, GMIP allows to model roles of participants in collaborative learning and interaction among them to achieve the learning goals. Thus, each interaction between two roles/participants is modeled as I_L events, defining which participant learns or supports the learning in a given interaction.

Although GMIP currently aims at describing CL, it can be used to model other forms of learning. Consider the case shown in Fig. 4 where three roles are defined. PeerTutor $\left(\right.$ Role $\left._{1}\right)$ teaches PeerTutee $\left(\right.$ Role $\left._{2}\right)$ and, from the behavior of PeerTutor, Observer $\left(\mathrm{Role}_{3}\right)$ learns how to teach others. As stated above, the basic unit of GMIP is a set of LGMs and an Interaction pattern. In the interaction ${ }_{12}$ each of PeerTutor and PeerTutee has its role's learning goal described as $\mathrm{LGM}_{1}$ and $\mathrm{LGM}_{2}$, respectively. On the other hand, in the interaction ${ }^{13}$, only Observer has the learning goal because PeerTutor is just observed and does not always need to be conscious of the Observer. The interaction pattern is an aggregation of the interactions between these roles. The I_L event decomposition tree (DT $1 \sim 3$ in Fig. 4) discussed in Section 3.1 fulfills a role to explain how each of the goals relates to the interaction pattern. In addition, an interaction pattern and some LGMs connected with the I_L event decomposition trees work as a generic model for learning and instruction. Even if the number of roles and interactions are increased, it can be modeled with additional LGMs and decomposition trees. On the other hand, in the case of one-to-one instruction, only an LGM and a decomposition tree are related with the interaction pattern because the learning goal of the instructor can be ignored, as in the example of the interaction between PeerTutor and Observer in Fig. 4.

Using this idea, we will show how to model CL as a formal product of the ID process with our proposed modeling framework through an example based on the theory 
"Peer tutoring" [6]. Figure 5 shows an example of collaborative learning model based on Peer tutoring. As mentioned above, in Peer tutoring, learners play two types of collaboration roles: the peer tutor role and the peer tutee role. The learning objective for each role can be described in the LGMs shown in Fig 5 (x).

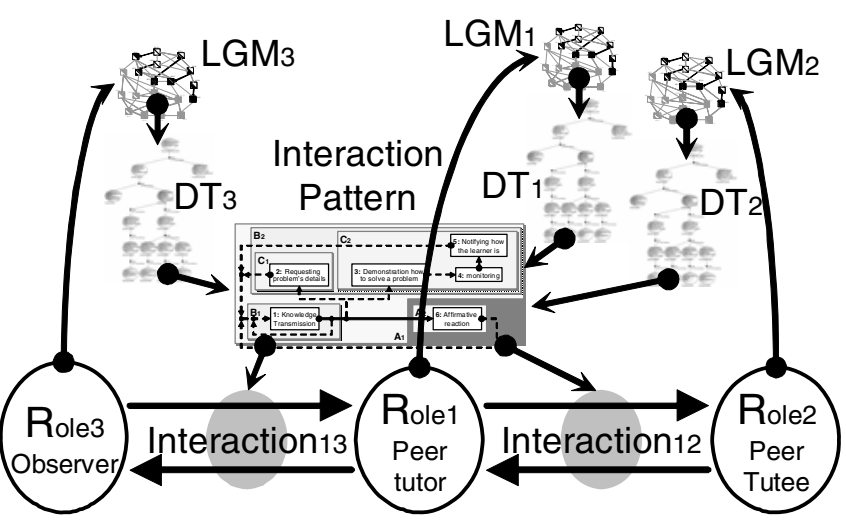

Fig. 4. An overview of the integrated model

Although there are some active paths in the LGMs (emphasized arrows in Fig. $5\left(\mathrm{x}_{1}\right.$, $\left.{ }_{2}\right)$ ), the essence is that the objective of peer tutor is Tuning and the one of peer tutee is Accretion as shown in Fig 5 ( $\left.\mathrm{x}^{\prime}\right)$.

These objectives are achieved by the activities of participants assigned to the roles, which are informing the topic to the peer tutee by the peer tutor, practice by the peer tutee, and guiding the practice by the peer tutor. These activities are defined as an interaction pattern shown as Fig. 5 (z), which is the one redrawn from Fig. 3 (b) in order to establish it to the I_L event decomposition trees (Fig. 5 (y)). The I_L event decomposition tree supplies links between the objective and the interaction pattern, and explains the design rationale of the link.

I_L event decomposition trees are constructed along the decomposition of learning objectives. Here the root of each decomposition tree is set as the objective defined by the LGM. This state (change) is decomposed into smaller-grain-sized ones with learning and instructional actions. Fig. 5 (y) illustrates a path of decomposition to a leaf I_L event in each I_L event decomposition tree. Each I_L event is decomposed into some I_L events or embodied in a much more concrete I_L event until the objectives are achieved by actions. The interaction pattern is the same as the sequence of the leaves of decomposition, which is interaction between the participants as a cycle of activities shown in Fig. $5(\mathrm{z})$. A cluster of the components in the interaction pattern corresponds to intermediate I_L event in the tree. For example, $A_{1}$ in Fig 5 (z) corresponds to both of $A_{1}$ in Fig $5(y)$, and each of them are decomposed into $B_{1}$ and $B_{2}$ in Fig 5 (y) because $A_{1}$ is composed of $B_{1}$ and $B_{2}$ in Fig 5 ( $\left.z^{\prime}\right)$.

As discussed in this section, through the line from LGM to Interaction pattern through I_L event decomposition tree, the design rationale of collaborative learning scenario can be revealed and maintained across the phases of instructional design. In addition, I_L event decomposition tree is helpful to assess the consistency between the learning objectives and the interactions. For example, there are other ways to achieve making the peer tutee meta-recognize his/her own understanding (Fig. $5\left(\mathrm{y}_{2}\right)$ $B_{2}$ ) than informing the peer tutee's performance (Fig. $\left.5\left(y_{2}\right)-5\right)$. An example is that the peer tutor demonstrates how the tutee solved the problem. In this case, although it is more difficult for the peer tutee to achieve, he/she can be trained in monitoring his/her own performance additionally. However, if the way is adopted, a problem occurs in 


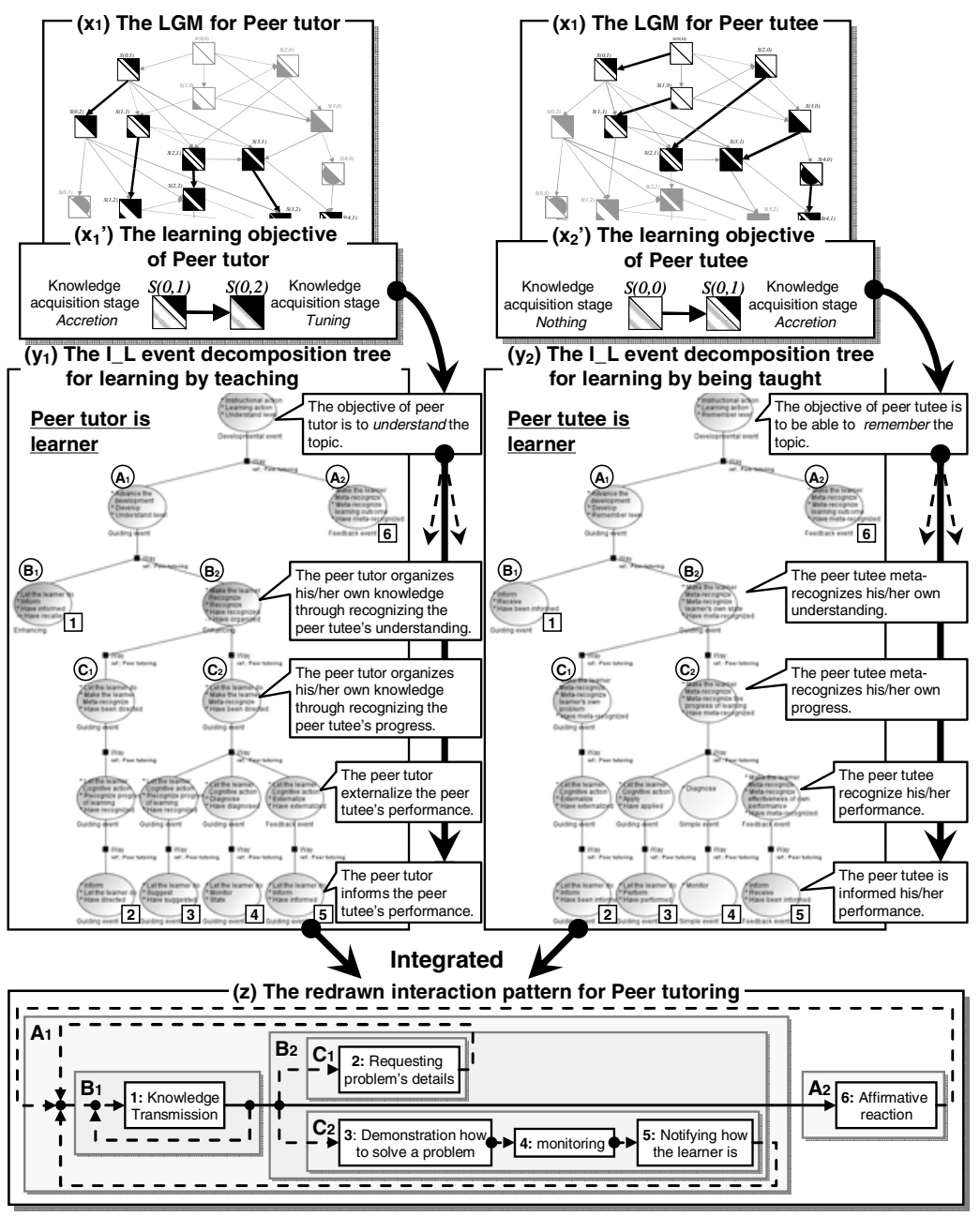

Fig. 5. An example of the integrated model

learning of peer tutor. In this scenario the peer tutor learns through diagnosing the peer tutee's performance and informing the result. The peer tutor cannot learn by just demonstrating again. Like this, in our proposed modeling framework, such inconsistency between collaboration roles can be identified easier than other modeling such as IMS LD.

If learning of PeerTutor is not intended in a learning session, this model can be considered to be the same as one-to-one instruction, in which PeerTutee learns through being taught by PeerTutor, neglecting GMIP and I_L event decomposition tree of PeerTutor. A set of a GMIP, an I_L event decomposition tree and an Interaction pattern is a basic unit for describing a learning session. Depending on the form of learning and on the number of roles that have intended learning objectives in the learning session, the composition of the integrated model of the session is decided. 
In conclusion, the presented framework allows for formally describing the product of the ID process for different forms of learning and, therefore, it helps to ensure the consistency of the product across the overall ID process and to manage the input/output of each phase of the ID process comprehensively.

\section{Conclusion}

The ID process is a complex task composed of many phases (analysis, design, development, implementation, and evaluation). To keep the consistency and the validity of the product (the course) in each phase, it is necessary to have a formal and semantically rich framework that allows for a better model of the product. Therefore, this paper discussed previous achievements on modeling individual and collaborative learning/instruction using ontologies, and how the accumulation of these past results together with a shared key concept to represent "learning" (I_L event) allow for the development of a framework that can describe formally learning and instructional scenarios. Such a description facilitates the sharing of the product of each ID phase and enables the systematic design of the course. To show the potential use of our framework, section 4 presented an example that covers the design phase of the ID process showing the creation of collaborative learning activities based on the Peer Tutoring theory. Due to space limitation, we could neither discuss the usability of our model in other ID phases nor present more details about the framework. However its potential benefits to support the ID process has been demonstrated.

The future direction of this study will expand the proposed modeling framework to tackle many other difficulties found in other phases of the ID process. For example, the analysis and development phases need much more detailed attributes in the context of learning and the implementation and evaluation phases require a mechanism for data collection and comparison of it to the design of course.

\section{References}

1. Amorim, R., Lama, M., Sánchez, E., Riera, A., Vila, X.: A Learning Design Ontology based on the IMS Specification. Journal of Educational Technology \& Society 9(1), 38-57 (2006)

2. Carr-Chellman, A.A., Hoadley, C.M.: Looking back and looking forward. Educational technology 44(3), 57-59 (2004)

3. Devedzic, V.: Semantic Web and Education. Springer Science Business Media, Heidelberg (2006)

4. Dicheva, D.: 4E Wiki (2008), http://o4e.iiscs.wssu.edu/xwiki/bin/view/Blog/Articles

5. Dick, W., Carey, L., Carey, J.O.: The systematic design of instruction, 5th edn. AddisonWesley Educational Publisher Inc., Reading (2001)

6. Endlsey, W.R.: Peer tutorial instruction. Ed Tech, Englewood Cliffs (1980)

7. Harrer, A.: An Approach to Organize Re-usability of Learning Designs and Collaboration Scripts of Various Granularities. In: Proc. of ICALT 2006 (2006) 
8. Hayashi, Y., Bourdeau, J., Mizoguchi, R.: Ontological Support for a Theory-Eclectic Approach to Instructional \& Learning Design. In: Nejdl, W., Tochtermann, K. (eds.) EC-TEL 2006. LNCS, vol. 4227, pp. 155-169. Springer, Heidelberg (2006)

9. Hayashi, Y., Mizoguchi, R., Bourdeau, J.: Structurization of Learning/Instructional Design Knowledge for Theory-aware Authoring systems. In: Woolf, B.P., Aïmeur, E., Nkambou, R., Lajoie, S. (eds.) ITS 2008. LNCS, vol. 5091, pp. 573-582. Springer, Heidelberg (2008)

10. Hernandez, D., Villasclaras, E.D., Asensio, J.I., Dimitriadis, Y.A., Retalis, S.: CSCL Scripting Patterns: Hierarchical Relationships and Applicability. In: Proc. of ICALT (2006)

11. IMS Global Learning Consortium, Inc.: IMS Learning Design. Version 1.0 (2003)

12. Inaba, A., Supnithi, T., Ikeda, M., Mizoguchi, R., Toyoda, J.: How Can We Form Effective Collaborative Learning Groups? In: Gauthier, G., VanLehn, K., Frasson, C. (eds.) ITS 2000. LNCS, vol. 1839, pp. 282-291. Springer, Heidelberg (2000)

13. Inaba, A., Ikeda, M., Mizoguchi, R.: What Learning Patterns are Effective for a Learner's Growth? In: Proc. of AIED 2003, pp. 219-226 (2003a)

14. Inaba, A., Ohkubo, R., Ikeda, M., Mizoguchi, R.: Models and Vocabulary to Represent Learner-to-Learner Interaction Process in Collaborative Learning. In: Proc. of ICCE 2003, pp. 1088-1096 (2003b)

15. Isotani, S., Mizoguchi, R.: An Integrated Framework for Fine-Grained Analysis and Design of Group Learning Activities. In: Proc. of ICCE 2006, pp. 193-200 (2006)

16. Koper, R.: An introduction to learning design. In: Koper, R., Tattersall, C. (eds.) Learning design A handbook on modeling and delivering networked education and training, pp. $3-$ 20. Springer, Heidelberg (2005)

17. Leshin, C.B., Pollock, J., Reigeluth, C.M.: Instructional Design Strategies \& Tactics. Educational Technology Publications, Englewood Cliffs (1992)

18. Mizoguchi, R., Bourdeau, J.: Using Ontological Engineering to Overcome Common AIED Problems. International Journal of Artificial Intelligence in Education 11(2), 107-121 (2000)

19. Mizoguchi, R., Hayashi, Y., Bourdeau, J.: Inside Theory-Aware \& Standards-Compliant Authoring System. In: Proc. of SWEL 2007, pp. 1-18 (2007)

20. Rawlings, A., van Rosmalen, P., Koper, R., Artacho, M., Lefrere, P.: Survey of Educational Modelling Languages (EMLs). CEN/ISSS WS/LT (2002),

http: / /www. cenorm. be/cenorm/businessdomains /

businessdomains/isss/activity/emlsurveyv1.pdf

21. Reigeluth, C.M.: What is instructional-design theory and how is it changing. In: Reigeluth, C.M. (ed.) Instructional-design theories and models A new paradigm of instructional theory, pp. 5-29. Lawrence Erlbaum Associates, Mahwah (1999)

22. Sloep, P., Hummel, H., Manderveld, J.: Basic design procedures for E-learning. In: Koper, R., Tattersall, C. (eds.) Learning design A handbook on modeling and delivering networked education and training, pp. 140-160. Springer, Heidelberg (2005) 